\title{
Fabrication of Advanced Electrode Materials for Supercapacitors
}

\author{
Ryan Poon, Igor Zhitomirsky \\ Department of Materials Science and Engineering, McMaster University \\ 1280 Main Street West, Hamilton, Ontario, Canada L8S 4L7 \\ poonry@mcmaster.ca; zhitom@mcmaster.ca
}

\section{Extended Abstract}

Electrochemical supercapacitors (ES) are currently under development for automotive and electronic applications due to their high power density and cyclic stability. Currently, several types of materials are under investigation for application in electrodes of ES, including metal oxides, conductive polymers, and high surface area carbon materials. In the design of ES cells, several factors should be taken into account, such as active mass, electrode material microstructure and specific capacitances of individual electrodes.

Manganese oxides are promising materials for ES electrodes due to high pseudocapacitance and low cost. The major problem of manganese oxides is low electronic and ionic conductivities, which result in poor electrochemical performance at high active mass loadings. The electronic conductivity of manganese oxide based electrodes can be improved by the use of conductive additives. However, the agglomeration of manganese oxide particles limits the electrolyte access to active surface. The goal of this investigation was the development of new techniques for the fabrication of advanced manganese oxide-carbon nanotube electrodes with enhanced performance at high active mass loadings and charge-discharge rates.

A new method has been developed to fabricate advanced electrodes, which is based on liquid-liquid extraction of synthesized nanoparticles from an aqueous phase to an organic phase. In this approach, the particle agglomeration during the drying can be avoided. The slurries of the manganese oxide particles and carbon nanotubes, dispersed in organic solvent, containing dissolved binders were used for the fabrication of electrodes. The approach was based on advanced extractor, which contained a galloyl group and hydrocarbon chain. Inspired by strong mussel adhesion to various surfaces in nature, investigations found catechol group is responsible for the strong adsorption. The structure of galloyl group contains three hydrophilic $\mathrm{OH}$ groups, and shows similar adsorption property as catechol by chelating or bridging mechanisms. The extractor molecules accumulated and aligned at the liquid-liquid interface, adsorbed on the particle surface and transferred particles into the organic phase to produce a slurry. $\mathrm{Mn}_{3} \mathrm{O}_{4}$-carbon nanotubes composite electrodes showed a capacitance of $2.63 \mathrm{~F} \mathrm{~cm}^{-2}$ at $2 \mathrm{mV} \mathrm{s}^{-1}$ and $0.95 \mathrm{~F} \mathrm{~cm}^{-2}$ at $100 \mathrm{mV} \mathrm{s}^{-1}$ at a mass loading of $30.4 \mathrm{mg} \mathrm{cm}^{-2}$. From literature, the areal capacitance of $\mathrm{Mn}_{3} \mathrm{O}_{4}$-carbon nanotubes electrode ranged from $0.14-0.6 \mathrm{~F} \mathrm{~cm}^{-2}$ at a mass loadings of 0.4-2 $\mathrm{mg} \mathrm{cm}^{-2}$. The $\mathrm{Mn}_{3} \mathrm{O}_{4}$ composite electrodes also exhibited near $90^{\circ}$ Nyquist plot with real part of impedance below 2 ohms which is critical for ES application. The results suggest the extraction technique reduces agglomeration and promotes electrolyte access to surface of electrode materials. In another approach, manganese dioxide nanoplatelets were prepared from $\mathrm{KMnO}_{4}$ solutions by a template-free method based on the use of new crystals, which combine properties of a reducing agent and a surfactant. The platelet structure allowed enhanced electrolyte access to the active material, and the microstructure was optimized for the fabrication of electrodes with enhanced performance at high active mass loading. The $\mathrm{MnO}_{2}$-carbon nanotube composite electrode showed a high capacitance of $8.95 \mathrm{~F} \mathrm{~cm}^{-2}$ at $2 \mathrm{mV} \mathrm{s}^{-1}$ at a mass loading of $61.2 \mathrm{mg} \mathrm{cm}^{-2}$. 